\title{
Indicadores de calidad en cápsula endoscópica y enteroscopia apoyada por dispositivos
}

\author{
Gerardo Blanco-Velasco* \\ Servicio de Endoscopia, Hospital de Especialidades, Centro Médico Nacional Siglo XXI, Instituto Mexicano del Seguro Social, Ciudad de México, \\ México
}

\section{Resumen}

Los índices de calidad en endoscopia son de gran utilidad para mejorar las condiciones y los resultados de los mismos. Debido a que la cápsula endoscópica y la enteroscopia son dos procedimientos endoscópicos relativamente nuevos los criterios de calidad no están tan establecidos como en otras áreas de la endoscopía. Durante los la DDW 2019 no se presentaron trabajos relacionados con calidad en enteroscopia o cápsula endoscópica, sin embargo, en los ESGE Days 2019 si se presentaron algunos estudios que fueron analizados en relación a la literatura existente.

Palabras clave: Calidad. Cápsula endoscópica. Cápsula enteroscopia.

\section{Introducción}

Los indicadores de calidad de la cápsula endoscópica (CE) y la enteroscopia apoyada por dispositivos (EAD) son distintos. Si bien es complicado identificar artículos que hablen específicamente de los indicadores de calidad en la EAD, se pueden adoptar los utilizados para los estudios endoscópicos en general. En el caso de la CE, existe mayor referencia en la literatura y los indicadores cambian de los del resto de los estudios endoscópicos.

\section{Trabajos realizados}

Durante los ESGE Days 2019, celebrados en Praga, República Checa, del 4 al 6 de abril del 2019 se presentaron 4 trabajos relacionados con calidad en CE y uno de EAD, mientras que en la Semana de Enfermedades Digestivas realizada en San Diego, California,
Estados Unidos del 18 al 21 de mayo del 2019, no se presentaron trabajos relacionados con este tema. A continuación se describirán los trabajos más importantes presentados en los ESGE Days 2019.

\section{Objetivo}

El objetivo de esta revisión será analizar los trabajos de calidad relacionados con la CE y EAD durante los ESGE Days 2019 y realizar una revisión de lo que existe relacionado con este tema en la literatura.

\section{Desarrollo}

\section{Enteroscopia apoyada por dispositivos}

Como se comentó en el párrafo anterior los principales indicadores de calidad en endoscopia se pueden adaptar a la enteroscopia apoyada por dispositivos. Sin

\section{Correspondencia:}


embargo, existe una guía reciente de la Sociedad Europea de Endoscopia Gastroeintestinal, conocida como ESGE por sus siglas en inglés que comenta los principales puntos para tener una enteroscopia de calidad ${ }^{1}$.

Divide los puntos en apartados como preprocedimiento, extensión del estudio, identificación de patología, manejo de patología, complicaciones, número de procedimientos y experiencia del paciente. Los índices de calidad se deben medir por cada 100 estudios realizados o de forma anual.

En el preprocedimiento se comenta que se debe tener indicación para EAD en más del 95\% de los procedimientos que realicemos. Las principales indicaciones de la enteroscopia son hemorragia de intestino delgado, enfermedad de Crohn, tumores y poliposis intestinales y enfermedad celiaca ${ }^{2}$. Otro índice es una adecuada preparación en más del $95 \%$ de los casos, siendo ayuno de 6 horas si es vía anterógrada y preparación similar a una colonoscopia si es vía retrógrada.

En cuanto a la extensión de la EAD, comenta que se debe realizar tatuaje de la máxima extensión en más del $80 \%$ de los casos y se debe poner la máxima extensión en el $80 \%$ de los reportes realizados.

En la identificación de la patología, se recomienda que la EAD debe de realizarse posterior a un estudio menos invasivo como es la CE o estudios de imagen. El hallazgo de la patología depende de la indicación del estudio y se debe identificar en $\geq 50 \%$ de los estudios realizados. También es importante foto-documentar las lesiones observadas en $\geq 95 \%$ de los reportes. Para el manejo de la patología, esta se debe tratar o tatuar en $\geq 95 \%$ y la terapéutica debe ser exitosa en $\geq 80 \%$ de los casos.

Las complicaciones se deben identificar $<5 \%$ de los procedimientos, siendo las más comunes, la pancreatitis $(0.2$ a $0.34 \%)$, hemorragia $(0.2$ a $0.8 \%$ ) y perforaciones $(0.1 \text { a } 2.9 \%)^{3}$.

En cuanto a la experiencia requerida para ser un enteroscopista experto no está bien documentada. Sin embargo, se sugiere que por lo menos haya realizado 15 EAD anterógradas y 35 retrógradas. Se desconoce el número de enteroscopias que debe realizar anualmente para mantener esta experiencia. El último punto que valora la guía es los síntomas del paciente post procedimiento, el cual debe estar asintomático en el $90 \%$ de los casos.

En cuanto a los estudios de calidad en EAD presentados en los ESGE Days 2019 y DDW 2019, solamente se presentó un trabajo. Este fue el realizado por Ismail, et al., en Dublín, Irlanda. En este estudio observaron la calidad de las EAD realizadas en el Tallaght University Hospital entre 2014 y 2017. En los reportes se identificó la indicación del estudio y la preparación utilizada en el $99.6 \%$ de los casos, la máxima inserción en el $95 \%$ y los hallazgos en el $100 \%{ }^{4}$. Si bien, faltan parámetros por medir en este estudio, los que no presentan en el reporte son de buena calidad.

\section{Cápsula endoscópica}

En cuanto a los índices de calidad para CE podemos encontrar mayor número de artículos publicados. La mayoría de ellos los dividen en preprocedimiento, intraprocedimiento y posprocedimiento ${ }^{1,5,6}$. De estas, nuevamente la guía de la ESGE es la más completa. El primer indicador es la presencia de indicación del estudio que tiene que estar en más del $95 \%$ de los estudios. Las indicaciones de cápsula endoscópica son: hemorragia de intestino delgado, anemia, sospecha o seguimiento de Enfermedad de Crohn, tumores, poliposis intestinal, diarrea crónica o enfermedad celiaca $^{7-13}$.

El segundo indicador es la preparación. Si bien existe debate acerca de la mejor preparación, esta debe de mencionarse en el reporte en $\geq 95 \%$ de los casos. Los laxantes y agentes antiespumosos no incrementan el rendimiento diagnóstico ni la tasa de visualización completa del intestino pero sí permiten una mejor visualización del intestino delgado ${ }^{14,15}$. Sin embargo, no se recomienda el uso generalizado de procinéticos ${ }^{15}$.

Otro indicador importante es la detección de lesiones, que aunque varía según la patología debe de ir acompañado de una imagen de la lesión en $\geq 95 \%$ de los casos. Otros indicadores que deben señalarse en el reporte es si hay o no visualización completa del intestino delgado ( $<80 \%$ de los casos), la velocidad de lectura (ideal 10 imágenes por segundo), recomendaciones para el tratamiento del paciente y las complicaciones asociadas al estudio de CE (ideal por debajo del $2 \%)$.

Todos los reportes deben realizarse utilizando la terminología estructurada de cápsula endoscópica (CEST por sus siglas en inglés) con la idea de que tanto el que escribe el reporte como el que lo lea manejen el mismo lenguaje ${ }^{16}$.

Por último para considerar a un endoscopista como experto para la lectura de $\mathrm{CE}$, se considera que este debe tener un entrenamiento de 30 a 50 CE leídas y realizar de 30 a $50 \mathrm{CE}$ anuales para mantener esta experiencia. 
Durante los ESGE Days 2019, se presentaron en Praga 4 trabajos relacionados con calidad en cápsula endoscópica, mientras que en la DDW 2019 no se presentó ninguno.

En uno de los trabajos presentados, se valoró si la presencia de imágenes opacas, borrosas y de bajo contrate afectaba para detectar tres tipos de lesiones: vasculares, inflamatorias y malignas. Observaron que las lesiones vasculares fueron las menos afectadas siendo diagnosticadas por el $90 \%$ de los lectores, mientras que las lesiones malignas fueron las más afectadas siendo diagnosticadas por solo el $50 \%$. Sugieren el incorporar en el software aditamentos que incrementen el contraste y disminuyan la opacidad de las lesiones para incrementar su detección ${ }^{17}$.

Otro estudio importante fue el valorar la calidad de los estudios de CE en España, siguiendo las recomendaciones de las guías de la ESGE. Se incluyó a 153 endoscopistas españoles expertos en CE. El 60.2\% contaban con un curso en CE. La velocidad de lectura promedio fue de 11 imágenes por segundo, con un $57.5 \%$ de los lectores con una velocidad de lectura por arriba de las 10 imágenes por segundo recomendadas. El $46.6 \%$ recomendaban dar laxantes previos al estudio de CE y $9.3 \%$ utilizaban procinéticos de manera cotidiana. El 54.1\% recomienda dar dieta líquida hasta dos horas después de la ingestión de la CE. Tan solo el $29.4 \%$ utilizaban la terminología CEST y el $7.8 \%$ no la conocían. Concluyen que en general se puede mejorar de forma significativa la lectura de CE en España $^{18}$.

\section{Conclusiones}

Tanto la EAD como la CE son dos procedimientos relativamente nuevos dentro de endoscopia, por este motivo existe menor evidencia en cuanto a su calidad con respecto a otros procedimientos endoscópicos. Sin embargo, la nueva guía de la ESGE ha permitido mostrar nuevos indicadores que mejoren la calidad de estos estudios.

\section{Bibliografía}

1. Spada C, Mcnamara D, Despott EJ, et al. Performance measures for small-bowel endoscopy: a Eurpean Society of Gastrointestinal Endoscopy (ESGE) quality improvement initiative. Endoscopy 2019;51:574-598.

2. Pennazio M, Spada C, Eliakim R, et al. Small-bowel capsule endoscopy and device-assisted enteroscopy for diagnosis and treatment of smaII- bowel disorders: European Society of Gastrointestinal Endoscopy (ESGE) clinical guideline. Endoscopy 2015;47:352-376.

3. Moeschler O, Mueller MK. Deep enteroscopy indications, diagnostic yield and complications. World J Gastroenterol 2015;21:1385-1393.

4. Ismail MS, O'Keefe A, Aylward CJ, et al. Future key performance indices for device assisted enteroscopy; what we can learn from current practice. Endoscopy 2019;51:S29.

5. Mitselos IV, Christodoulou DK. What defines quality in small bowel capsule endoscopy. Ann Transl Med 2018;6:260.

6. Shim KN, Jeon SR, Jang HJ, et al. Quality indicators for small bowel capsule endoscopy. Clin Endosc 2017;50:148-160.

7. Raju GS, Gerson L, Das A et al. American Gastroenterological Association (AGA) Institute medical position statement on obscure gastrointestinal bleeding. Gastroenterology 2007;133:1694-1696.

8. Riccioni ME, Urgesi R, Spada C et al. Unexplained iron deficiency anaemia: Is it worthwhile to perform capsule endoscopy? Dig Liver Dis 2010; 42.560-566

9. Tukey M, Pleskow D, Legnani P et al. The utility of capsule endoscopy in patients with suspected Crohn's disease. Am J Gastroenterol 2009;104:2734-2739.

10. Rondonotti E, Pennazio M, Toth E, et al. Small-bowel neoplasms in patients undergoing video capsule endoscopy: a multicenter European study. Endoscopy 2008;40:488-495.

11. Brown G, Fraser C, Schofield G, et al. Video capsule endoscopy in peutz-jeghers síndrome: a blinded comparison with barium follow-through for detection of small-bowel polyps. Endoscopy 2006;38:385-390.

12. Petroniene R, Dubcenco E, Baker JP, et al. Given capsule endoscopy in celiac disease: evaluation of diagnostic accuracy and interobserver agreement. Am J Gastroenterol 2005;100:177-185.

13. Cheung DY, Kim JS, Shim KN, et al. The usefulness of capsule endoscopy for small bowel tumors. Clin Endosc 2016;49:21-25.

14. Gkolfakis P, Tziatzios G, Dimitriadis GD et al. Meta-analysis of randomized controlled trials challenging the usefulness of purgative preparation before small-bowel video capsule endoscopy. Endoscopy 2018; 50: 671-668.

15. Kotwal VS, Attar BM, Gupta S et al. Should bowel preparation, antifoaming agents, or prokinetics be used before video capsule endoscopy? A systematic review and meta-analysis. Eur J Gastroenterol Hepatol 2014; 26: 137-145.

16. Korman LY, Delvaux M, Gay G et al. Capsule endoscopy structured terminology (CEST): proposal of a standardized and structured terminology for reporting capsule endoscopy procedures. Endoscopy 2005; 37: 951-959.

17. Yung DE, Plevris JN, Koulaouzidis A. Poor quality of capsule endoscopy images has a significant negative effect on the diagnosis of small bowel malignancy. Endoscopy 2019;51:S8-S9.

18. C Carretero, Lujan M, Sanjuan M, et al. Results of a spanish national survey of capsule endoscopy. Endoscopy 2019;51:S180. 